\title{
Summary of Deflections from Fully Assembled EC Structure Finite Element Model
}

\author{
Bob Wands
}

\section{Introduction}

The purpose of this note is to summarize the deflections in the fully assembled $E C O H$ and $M H$ module arrays as calculated by a superelement finite element model. These results are for the case where the $\mathrm{OH}$ modules are fully connected at four locations on the inner radius and four locations on the outer radius. Both the $\mathrm{OH}$ and $\mathrm{MH}$ modules are modeled as skins and endplates (and in the case of $\mathrm{OH}$, internal structural plates) only. The $\mathrm{OH}$ module has a skin thickness of $1 / 16$ th in.

Fig. 1 shows the fully assembled model and the coordinate system used to express deflections. The deflections at the inner and outer radius at both the front (nearest the collision center) and the back of the module assemblies are given in Tables I-IV. A summary of the deflections is given in Figs. 2-4. 
Table I. Deflections at Front of OH Assembly

\begin{tabular}{|c|c|c|c|c|c|c|}
\hline \multirow[t]{3}{*}{ Theta } & \multicolumn{3}{|c|}{ Inner } & \multicolumn{3}{|c|}{ Outer } \\
\hline & & Radius & & & Radius & \\
\hline & UX & UY & $\mathrm{UZ}$ & UX & UY & $\mathrm{UZ}$ \\
\hline 0.0 & 0.0 & -.029 & .007 & 0.0 & -.019 & -.007 \\
\hline 22.5 & -.002 & -.026 & .007 & -.003 & -.015 & -.007 \\
\hline 45.0 & $<.001$ & -.018 & .007 & -.002 & -.003 & -.004 \\
\hline 67.5 & -.007 & -.012 & .015 & .007 & .003 & .005 \\
\hline 90.0 & .007 & -.011 & .039 & .005 & .001 & .042 \\
\hline 112.5 & .006 & -.010 & .050 & .007 & -.002 & .064 \\
\hline 135 & .006 & -.010 & .070 & .012 & -.007 & .064 \\
\hline 157.5 & -.001 & -.021 & .086 & .001 & -.022 & .083 \\
\hline 180.0 & 0.0 & -.024 & .083 & 0.0 & -.021 & .089 \\
\hline
\end{tabular}




\section{Table II. Deflections at Back of OH Arch}

\begin{tabular}{|c|c|c|c|c|c|c|}
\hline \multirow[t]{2}{*}{ Theta } & \multicolumn{3}{|c|}{$\begin{array}{l}\text { Inner } \\
\text { Radius }\end{array}$} & \multicolumn{3}{|c|}{$\begin{array}{l}\text { Outer } \\
\text { Radius }\end{array}$} \\
\hline & $U X$ & UY & $\mathrm{UZ}$ & UX & UY & $\mathrm{UZ}$ \\
\hline 0.0 & 0.0 & -.063 & .011 & 0.0 & -.047 & -.011 \\
\hline 22.5 & -.006 & -.060 & .012 & -.005 & -.041 & -.011 \\
\hline 45.0 & -.006 & -.050 & .016 & -.005 & -.027 & -.007 \\
\hline 67.5 & -.002 & -.041 & .025 & -.004 & -.040 & -.007 \\
\hline 90.0 & .008 & -.036 & .040 & .007 & -.024 & .041 \\
\hline 112.5 & .008 & -.034 & .056 & .008 & -.026 & .061 \\
\hline 135 & .005 & -.031 & .072 & .010 & -.029 & .068 \\
\hline 157.5 & $<.001$ & -.039 & .085 & .002 & -.040 & .083 \\
\hline 180.0 & 0.0 & -.041 & .084 & 0.0 & -.037 & .090 \\
\hline
\end{tabular}


Table III. Deflections at Front of MH Assembly

\begin{tabular}{|c|c|c|c|c|c|c|}
\hline \multirow{3}{*}{ Theta } & \multicolumn{3}{|c|}{ Inner } & \multicolumn{3}{|c|}{ Outer } \\
\hline & & Radius & & & Radius & \\
\hline & UX & UY & $\mathrm{UZ}$ & UX & UY & $\mathrm{UZ}$ \\
\hline 0.0 & 0.0 & -.032 & .003 & 0.0 & -.032 & -.040 \\
\hline 11.25 & .002 & -.031 & 0.0 & .003 & -.032 & -.030 \\
\hline 33.75 & .006 & -.033 & $<.001$ & .007 & -.035 & -.017 \\
\hline 56.25 & .007 & -.037 & .005 & .010 & -.038 & -.006 \\
\hline 78.75 & .005 & -.039 & .012 & .004 & -.044 & .009 \\
\hline 101.25 & .002 & -.042 & .021 & .001 & -.045 & .024 \\
\hline 123.75 & $<.001$ & -.045 & .028 & $<.001$ & -.045 & .039 \\
\hline 146.25 & $<.001$ & -.048 & .034 & .001 & -.047 & .050 \\
\hline 168.75 & $<.001$ & -.049 & .038 & $<.001$ & -.048 & .056 \\
\hline 180.00 & 0.0 & -.049 & .038 & 0.0 & -.049 & .056 \\
\hline
\end{tabular}


Table IV. Deflections at Back of MH Assembly

\begin{tabular}{|c|c|c|c|c|c|c|}
\hline \multirow[t]{3}{*}{ Theta } & \multirow{2}{*}{\multicolumn{3}{|c|}{$\begin{array}{l}\text { Inner } \\
\text { Radius }\end{array}$}} & \multirow{2}{*}{\multicolumn{3}{|c|}{$\begin{array}{l}\text { Outer } \\
\text { Radius }\end{array}$}} \\
\hline & & & & & & \\
\hline & UX & UY & UZ & UX & UY & UZ \\
\hline 0.0 & 0.0 & -.07 & -.005 & 0.0 & -.070 & .034 \\
\hline 11.25 & .002 & -.066 & -.006 & .003 & -.070 & -.020 \\
\hline 33.75 & .006 & -.069 & -.003 & .008 & -.070 & -.014 \\
\hline 56.25 & .006 & -.074 & .004 & .010 & -.074 & -.003 \\
\hline 78.75 & .005 & -.076 & .012 & .004 & -.080 & .009 \\
\hline 101.25 & .002 & -.079 & .020 & .001 & -.081 & .024 \\
\hline 123.75 & .006 & -.082 & .030 & $<.001$ & -.081 & .037 \\
\hline 146.25 & $<.001$ & -.084 & .036 & .001 & -.083 & .047 \\
\hline 168.75 & $<.001$ & -.085 & .039 & $<.001$ & -.084 & .053 \\
\hline 180.00 & 0.0 & -.085 & .040 & 0.0 & -.085 & .054 \\
\hline
\end{tabular}




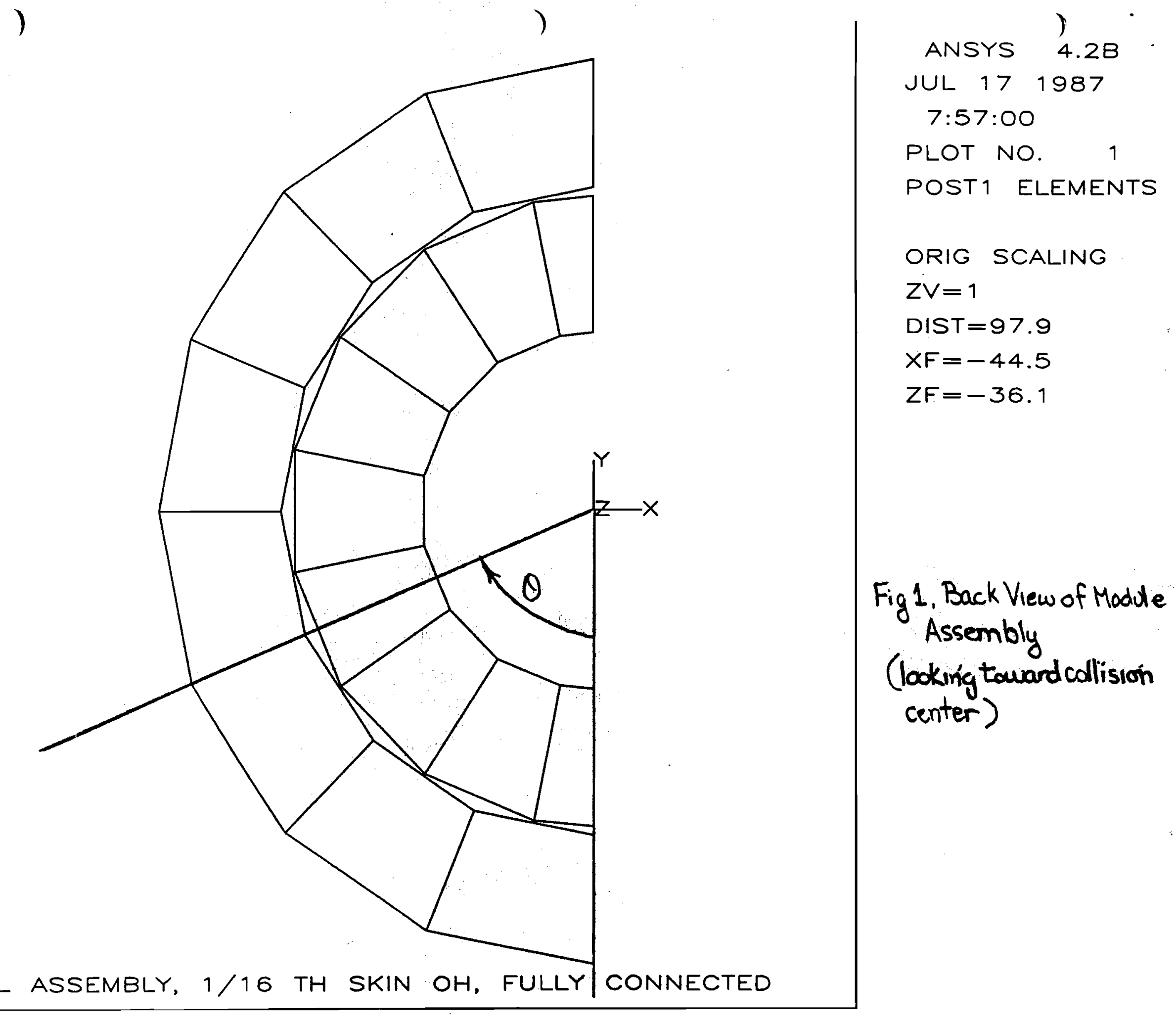




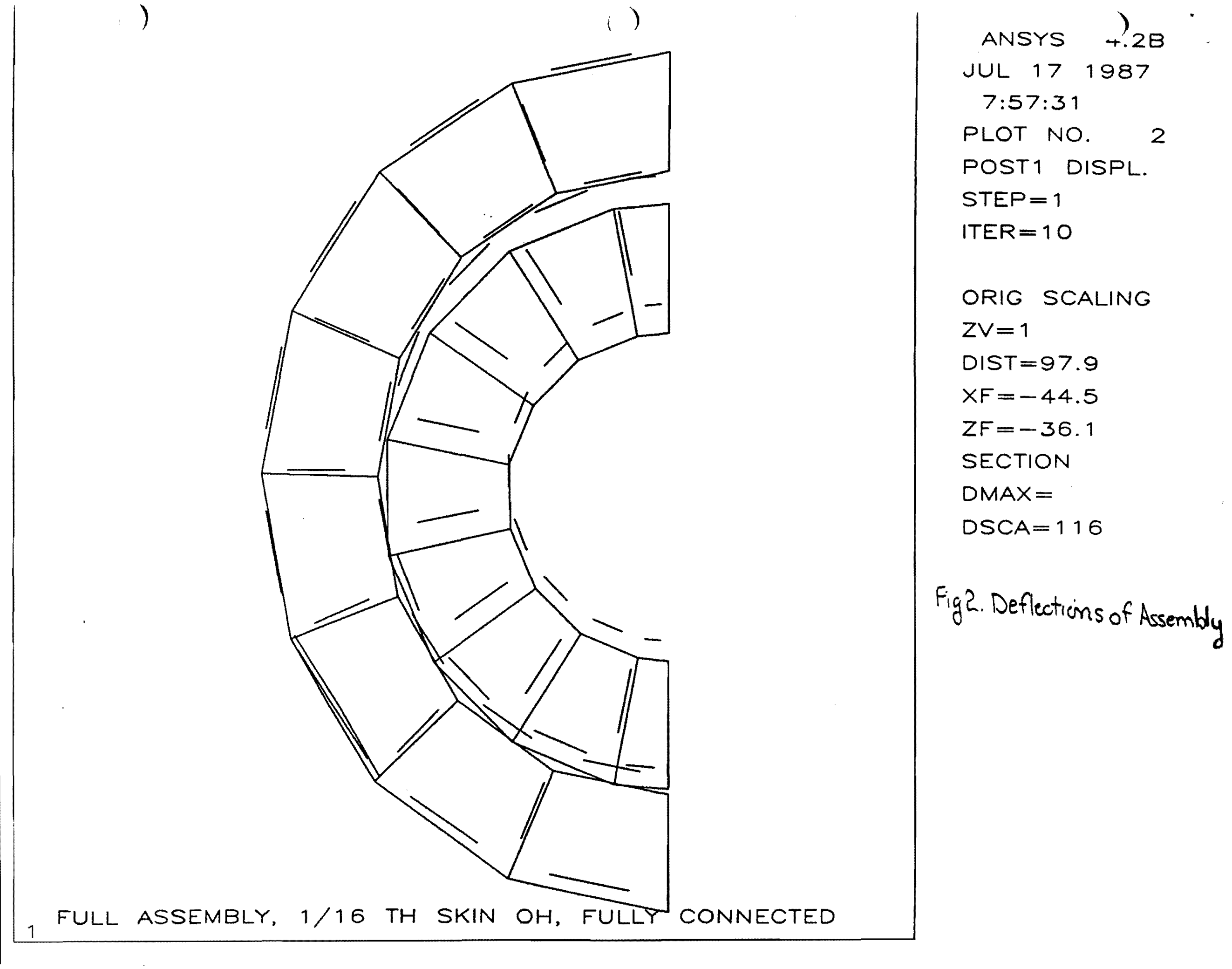




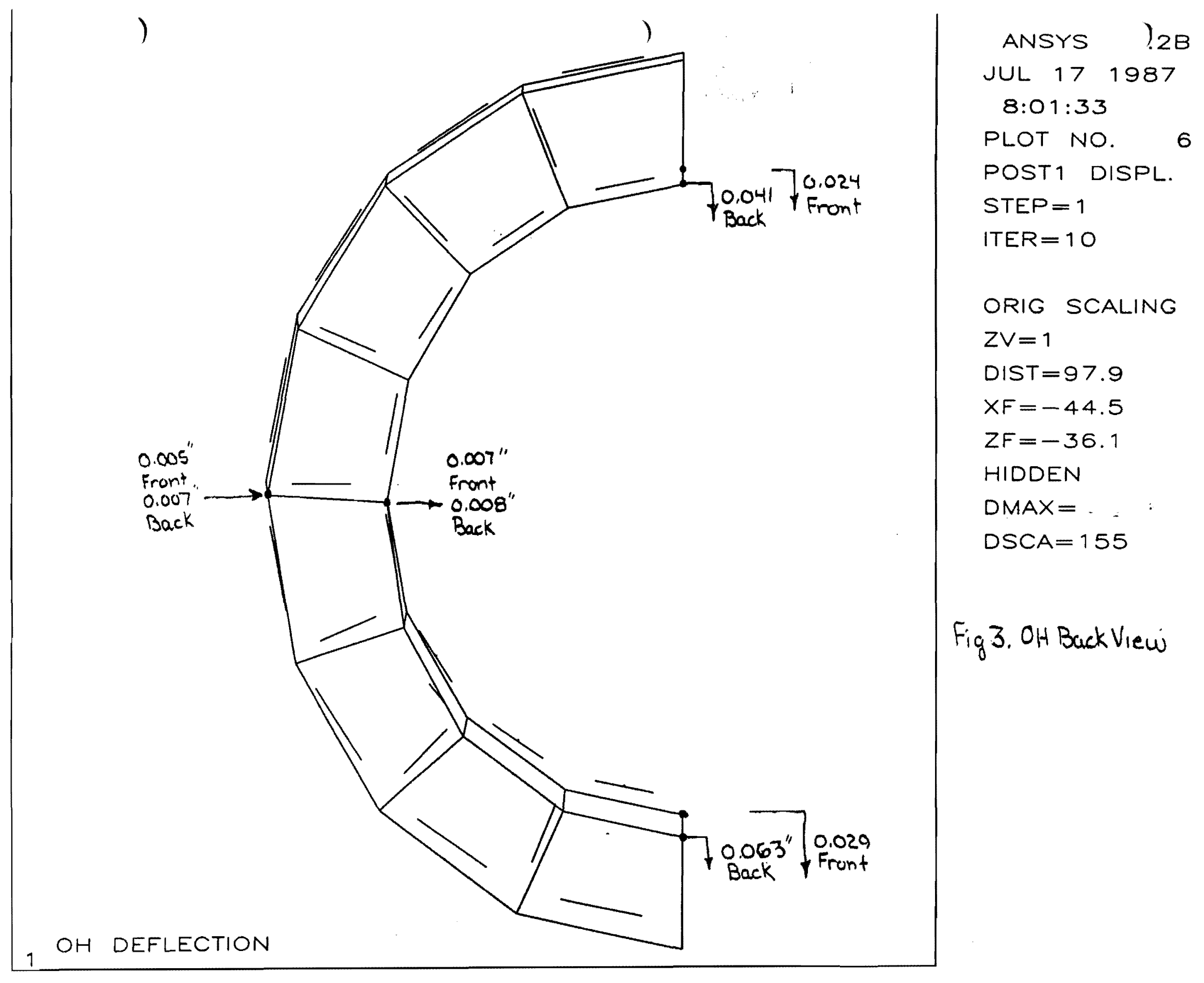




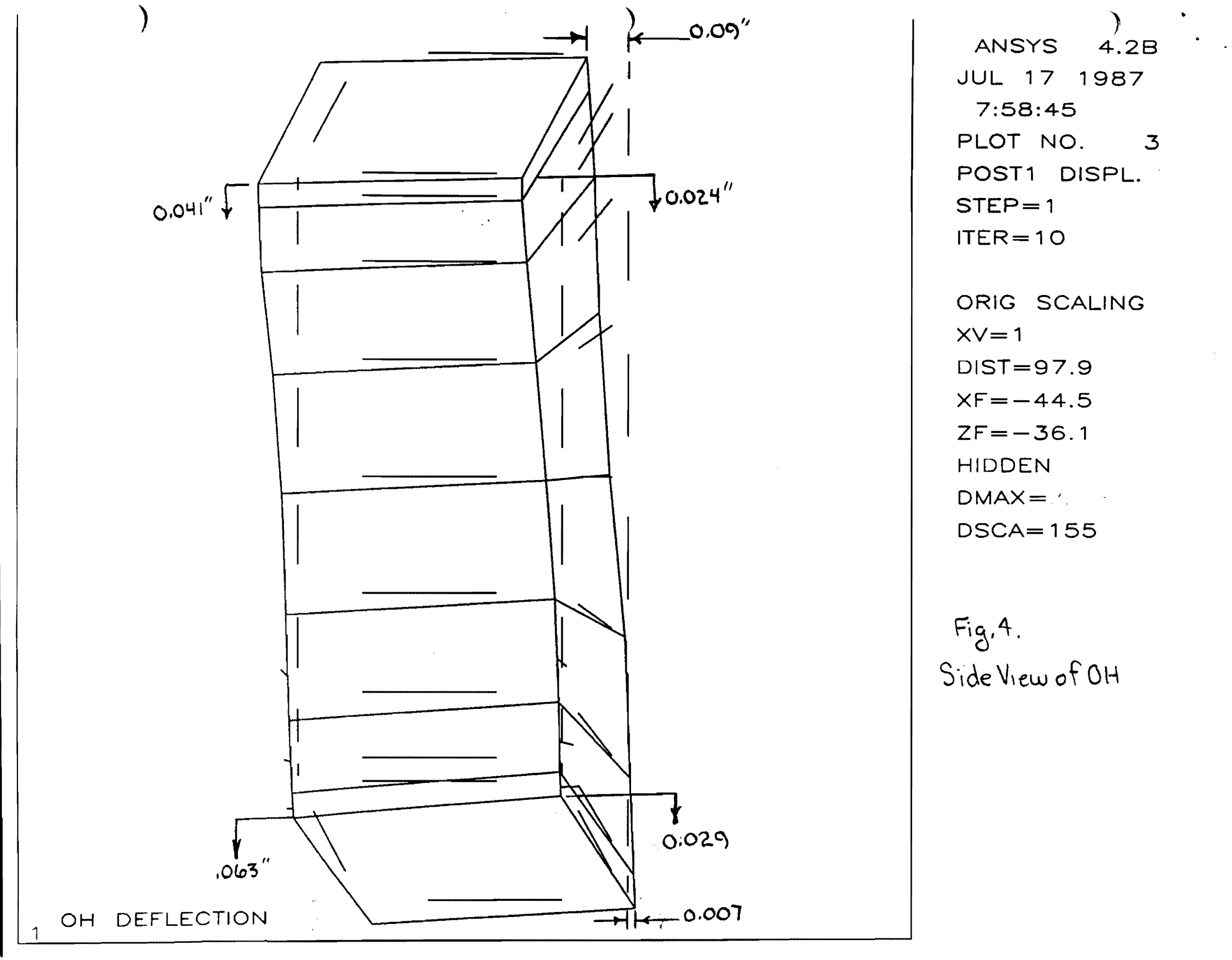




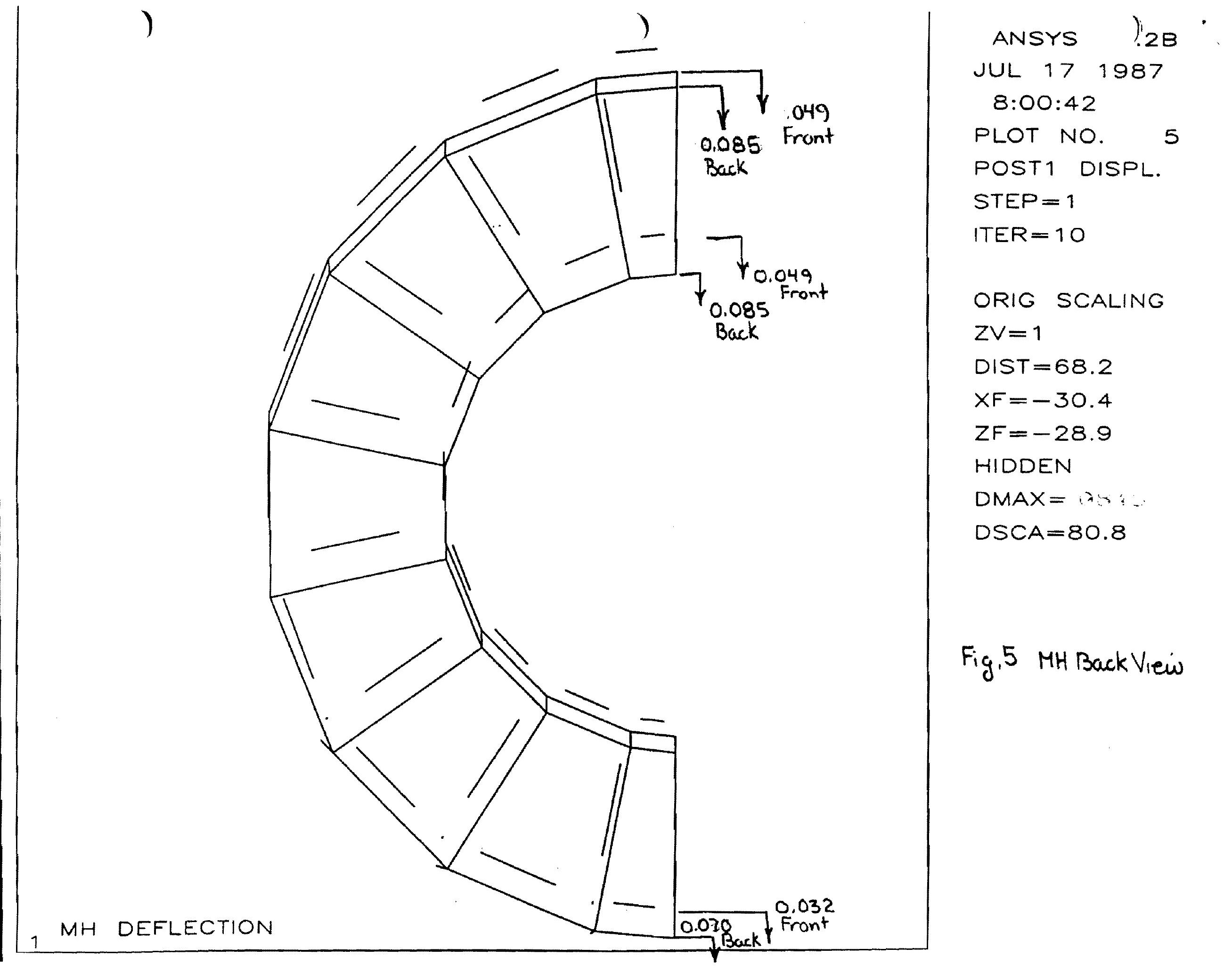

